\title{
Réseaux sociaux et les capacités financières des filles employées de maison à Bobo-Dioulasso
}

Gisele Kaboré

Population Council

Follow this and additional works at: https://knowledgecommons.popcouncil.org/departments_sbsr-pgy

Part of the Demography, Population, and Ecology Commons, Family, Life Course, and Society Commons, Gender and Sexuality Commons, International Public Health Commons, Medicine and Health Commons, and the Women's Health Commons How does access to this work benefit you? Let us know!

\section{Recommended Citation}

Kaboré, Gisele. 2012. "Réseaux sociaux et les capacités financières des filles employées de maison à Bobo-Dioulasso." Ouagadougou: Population Council. 
RÉSEAUX SOCIAUX ET LES CAPACITÉS FINANCIĖRES DES

FILLES EMPLOYÉES DE MAISON

À BOBO-DIOULASSO

PROGRAMME FILLES ÉVEILLÉES

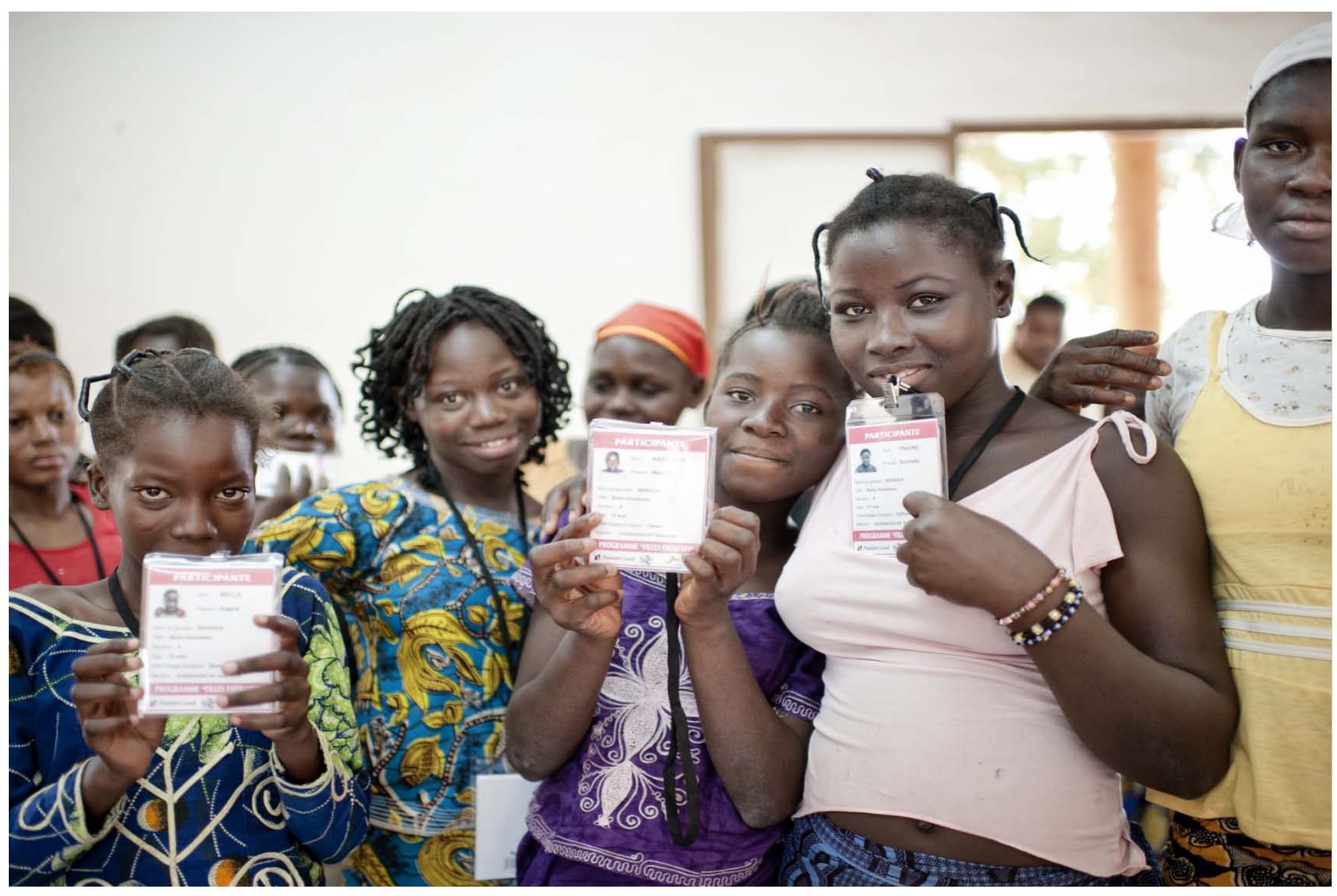




\title{
(2) Population Council
}

Le Population Council s'attelle aux questions cruciales de la santé et du développement - qu'il s'agisse d'endiguer la progression du VIH, d'améliorer la santé reproductive et d'offrir aux jeunes la promesse d'une vie satisfaisante et productive. Par ses travaux de recherche en biomédecine, en sciences sociales et en santé publique dans 50 pays, il s'efforce d'apporter avec ses partenaires des solutions qui conduisent à des politiques, des programmes et des technologies plus efficaces, pour améliorer la vie partout dans le monde. Fondé en 1952, le Population Council est une organisation non gouvernementale à but non lucratif. II siège à New York sous la conduite d'un conseil d'administration international.

\author{
Population Council \\ One Dag Hammarskjold Plaza \\ New York, NY 10017 \\ Population Council \\ 01 BP 6250 \\ Ouagadougou 01 \\ Burkina Faso
}

www.popcouncil.org

Indication de source suggeré: Gisele Kabore. 2012. "Réseaux Sociaux et les Capacités Financières des Filles Employées de Maison à Bobo-Dioulasso." Ouagadougou: Population Council.

Photo courtoisement offerte par Ollivier Girard.

(c) 2012. The Population Council, Inc. 


\section{TABLE DES MATIĖRES}

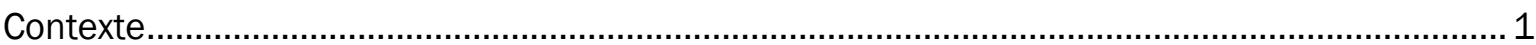

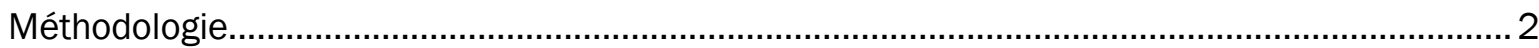

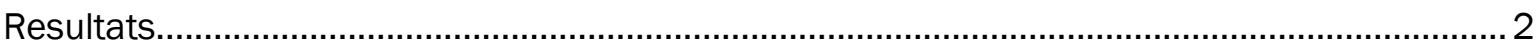

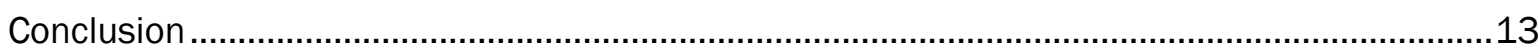

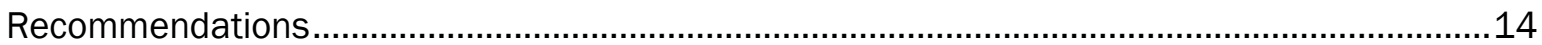


Depuis plus d'une année, le Population Council assure la mise en œuvre d'un projet ciblant les travailleuses domestiques dans les zones urbaines au Burkina. A Bobo-Dioulasso, ces filles proviennent d'origines diverses et sont employées comme filles de ménage, dans les restaurants ou les marchés. La recherche formative faite par le Council a montré les principales raisons pour la migration des adolescentes sont : chercher un emploi, chercher de l'argent pour préparer leur avenir, échapper à un mariage précoce ou forcé, etc. L'arrivée des filles est généralement saisonnière. Elles migrent généralement vers les grandes villes à la fin de la saison pluvieuse (en octobre ou novembre) et y travaillent pendant environ 8 mois et elles retournent au village au début de la saison pluvieuse (juin-juillet) pour aider leurs parents dans les travaux champêtres. Certaines d'entre elles restent au village pour préparer leur mariage et c'est la fin de la migration pour elles.

Une première cohorte de filles a participé au programme "Filles Eveillées " à Bobo-Dioulasso. Plusieurs actions ont été menées dans ce cadre notamment le renforcement des compétences dans quatre domaines : compétences de vie courante, capacités financières, hygiène et santé et santé de la reproduction. Les groupes de filles se rencontrent une fois par semaine pendant deux (2) heures pour une période de huit (8) mois et se focalisent sur le développement de leurs compétences dans les quatre domaines ci-dessus cités. Le programme va donc au-delà de la simple augmentation des connaissances des filles.

Cette étude qualitative vient en complément aux enquêtes quantitatives de base et de fin dans le but de mieux connaitre l'environnement de vie des filles. L'objectif de cette étude est d'avoir une meilleure connaissance des réseaux sociaux des filles-soit avec les personnes dans sa nouvelle communauté soit avec des personnes dans son village à travers la technologie-depuis leurs zones d'origine, sachant que la phase de transit n'est pas bien comprise (Temin, Montgomery, Engebretsen, forthcoming) mais que ça peut influencer le séjour de la fille en ville. Les filles migrantes manquent de réseaux sociaux par rapport aux filles non-migrantes. Les avantages de l'appartenance à un groupe sont clairs: la recherche dans le KwaZulu Natal, en Afrique du Sud a révélé que l'appartenance au groupe des filles a eu pour effet de retarder les premiers rapports sexuels chez les filles, de réduire l'incidence des rapports sexuels non consentent, et a augmenté l'utilisation du préservatif contrairement aux filles qui n'avaient aucune affiliation au programme. Les filles dans les groupes étaient onze fois plus susceptibles d'utiliser un préservatif lors du dernier rapport sexuel que les filles qui n'étaient pas dans un groupe (Hallman 2008).

Cette étude a pour objectif de cerner les capacités et les modes de gestion financières des filles, et de mesurer la connaissance du risque selon les circuits d'épargne. Les filles migrantes lorsqu'elles arrivent en ville sont économiquement actives. Elles ont des objectifs financiers mais manquent cependant de compétences sur les capacités financières pour leur permettre d'atteindre leur but. Cette situation est aggravée par le fait que la plupart de ces filles sont analphabètes. Ce volet s'avère donc d'une importance capitale pour ces filles et le programme "Filles Eveillées" se donne pour mission de développer les capacités des filles à épargner, à budgétiser, à développer des plans financiers. 


\section{METHODOLOGIE}

Cette enquête qualitative a utilisé l'approche des groupes de discussions dirigées (focus group). Elle a permis de mieux appréhender les réseaux sociaux des filles, de comprendre leurs problèmes, leurs capacités et leurs modes de gestion financière. L'approche des groupes de discussions dirigées a été privilégiée par rapport aux entretiens individuels approfondis pour amener les filles à échanger autour de leurs expériences communes et pouvoir dégager les tendances.

La population cible est composée des filles ayant participé dans la première phase du programme "Filles Eveillées" à Bobo-Dioulasso. Les zones concernées sont: secteur 2 (Diarradougou), secteur 4 (Kôko), secteur 8 (Sikassocira), secteur 9/10 (Accarville), et secteur 17 (Sarfalao).

La technique de collecte des données s'est reposée sur l'organisation de focus avec l'appui des mentors et des chargés de programme de l'association Tié. Ces focus ont regroupé en moyenne 9 participantes dont l'âge varie entre 11 et 17 ans. Au total 5 groupes ont été constitués pour les focus et on a enregistré une participation de 49 filles. Ces focus ayant été réalisés pendant la saison des pluies, on a pu regrouper que les filles qui sont restées en ville.

Tous les focus group ont été enregistrés et transcrits. Les données ont été saisies et traitées manuellement selon l'analyse croisée par contenu.

\section{RESULTATS}

\section{Caractéristiques des Filles Enquêtées}

Les filles ayant participé aux entretiens ont un âge variant entre 11 et 17 ans avec un âge moyen de 13 ans.

Les régions d'origine de la plupart des filles sont la région du SudOuest et de la Boucle du Mouhoun du Burkina Faso (Safané, Diébougou). Quelques filles (une minorité) sont originaires des Hauts-Bassins, de la région des Cascades et des régions du Nord, Centre-Nord et Centre-Est du Burkina Faso (Yako, Kaya, Koupela). Quelques-unes sont arrivées à Bobo-Dioulasso, en provenance du Mali (régions de Mopti, Sikasso, situées juste à quelques kilomètres après la

Figure 1 : Carte du Burkina Faso

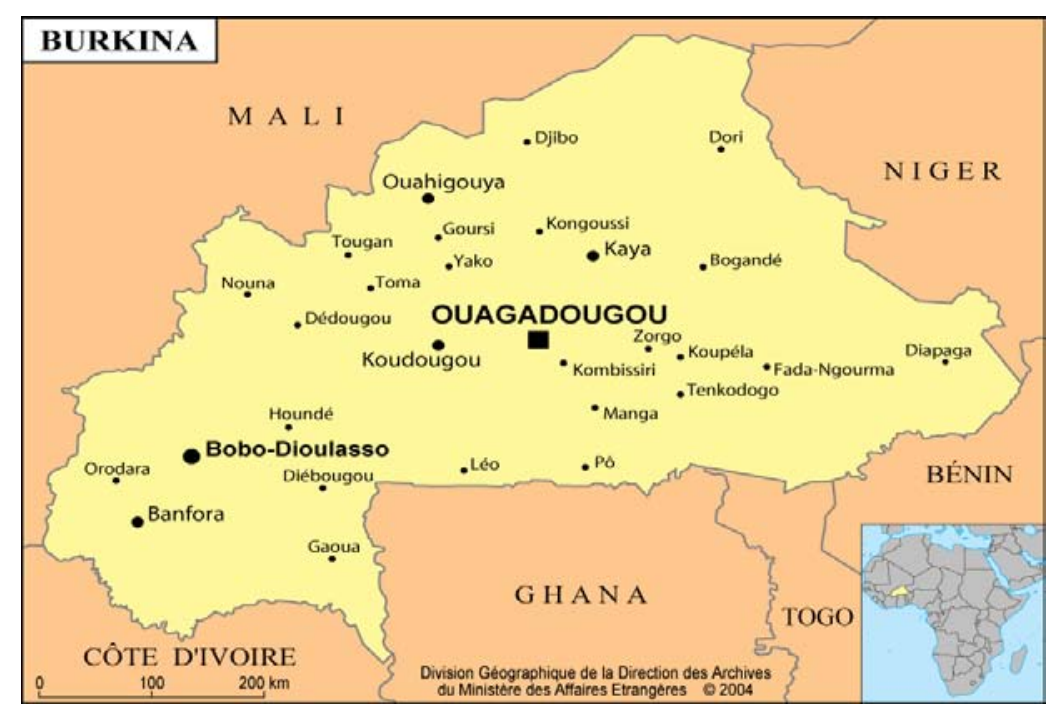
frontière) ou de Bouaké en république de Côte d'Ivoire. 
Plus de la moitié ont un niveau d'étude du primaire, le niveau le plus élevé étant la classe CM2 (cours moyen deuxième année). Cet effectif important de filles scolarisées est dû en partie au programme "Filles Eveillées ", qui paie les frais scolaires pour les participantes intéressées.

Tableau 1 : Le niveau d'étude des filles rencontrées

\begin{tabular}{lcc}
\hline Niveau d'instruction & Nombre de filles & Pourcentage (\%) \\
Non-instruit & 23 & 46,9 \\
Cours préparatoires 1 et 2 & 8 & 16,3 \\
\hline Cours élémentaires 1 et 2 & 5 & 10,2 \\
\hline Cours moyens 1 et 2 & 13 & 26,5 \\
\hline Total & 49 & 100,0 \\
\hline
\end{tabular}

NB : Le programme «Filles Eveillées » a inscrit les filles qui sont intéressées au cours du soir

\section{Réseaux Sociaux des Filles}

\section{Réseaux Sociaux de Transit}

De l'avis de la plupart des filles ayant participé au focus, les départs de leur village s'effectuent généralement avec les parents. Les trajectoires sont linéaires de leurs sites de départ à la ville (Bobo-Dioulasso). En général, il n'y a pas de site de transit pour les migrations internes comme le reconnaissent l'ensemble des filles.

"Du village, le véhicule les amène directement à la gare. De la gare, elles se reposent un peu, avant d'emprunter un taxi pour aller là où elles veulent. "

-FILLE DE 16 ANS, 1 AN DE TRAVAIL, ARRIVEE EN 2007 DE SIKASSO AU MALI.

Cependant, des escales sont effectuées au cas où le trajet est assez long comme l'expliquent quelques filles en provenance de la Côte d'Ivoire ou du Mali.

"La fille peut descendre dans une autre ville avant d'arriver à Bobo. De fois, il peut arriver de faire deux jours en route avant d'arriver à la maison, parce qu'on peut changer de véhicule"

-FILLE DE 13 ANS, 3 ANS DE TRAVAIL, ORIGINAIRE D’ABIDJAN, ARRIVEE EN 2003.

Au cours du voyage qui se fait sans transit, la grande majorité des filles arrivent à Bobo, avec des membres de la famille ou avec des camarades du village surtout lorsqu'il s'agit de la première migration. Une fille explique :

"Au cours du voyage, les filles dépendent des parents comme la tante, la grande mère, la mère ou le petit frère du père "

-FILLE DE 12 ANS ORIGINAIRE DE SAFANE, 1 AN DE TRAVAIL.

D'autres filles arrivent en ville en compagnie de leurs tuteurs ou de l'employeur. En effet, le recrutement des filles peut se faire directement par les employeurs au cours de leur séjour dans les villages d'origine des filles, comme l'attestent ces propos: 
"Il y a des filles qui viennent avec leurs tuteurs. Donc elles dépendent de lui "

-FILLE DE 16 ANS, ORIGINAIRE DU SUD-OUEST (DIEBOUGOU), ARRIVEE EN 2007, 3 ANS D'ACTIVITE

On note aussi que les voyages peuvent s'effectuer sans accompagnateurs. Cette situation est régulière chez les filles d'un âge avancé qui ont eu l'occasion d'effectuer plusieurs voyages dans la ville. Au cours du voyage, les filles avec ou sans accompagnateurs dorment dans les salles d'attente des gares dans l'attente de la poursuite du voyage. Une fille explique :

"Certaines filles viennent d'elles-mêmes. Comme ça en route, elles peuvent faire escale et demander à dormir quelque part- en gare- avant de continuer le voyage (si la route est longue ou si le véhicule tombe en panne)",

-FILLE DE 13 ANS, EN PROVENANCE D’ABIDJAN, ARRIVEE EN 2003 ; 3 ANS D'ACTIVITE

Au cours de ces entrevues, les filles n'ont pas fait cas de la traite des enfants.

\section{Attitudes en cas de problèmes}

De l'avis de la plupart des filles, les voyages se font sans difficultés. Les quelques problèmes qu'elles rencontrent se rapportent au manque d'argent, ou certaines maladies comme le paludisme qui surviennent en cours de voyage. Les résultats montrent que lorsque les filles quittent le village et arrivent en ville, les personnes chez qui elles s'hébergent sont un parent. En cas de problèmes, les personnes de recours sont les parents avec qui elles ont effectué le voyage (tante, grande-sœur, oncle). Au cas où les filles sont seules, elles font recours à leurs compères, aux chauffeurs ou à certaines personnes originaires du même village qu'elles en attendant que les parents ou tuteurs en ville ne viennent rembourser les sommes empruntées. En quittant le village, les filles prennent le soin de prendre avec elles les adresses des parents et elles peuvent appeler en cas de problème. Ce sont les réseaux sociaux et les systèmes indigènes qui protègent la fille quand elle arrive.

"En général, si on a des problèmes on fait recours aux parents ou les personnes qui nous ont fait venir en ville"

-FILLE DE 15 ANS ORIGINAIRE DE YAKO

"La personne qui la fait venir du village l'aide à résoudre leur problème "

-FILLE DE 12 ANS, ORIGINAIRE DE KOTOURA, ARRIVEE EN 2010

L'expédition peut être de courte durée au cas où la personne dont les filles dépendent n'arrive pas à les aider à résoudre ses problèmes. Une fille originaire de Diébougou l'atteste comme suit :

"Une tante aussi peut m'aider. Si c'est difficile, je retourne au village "

-FILLE DE 16 ANS, ORIGINAIRE DE DIEBOUGOU.

Les difficultés que peuvent rencontrer les filles sont les cas de maladies graves nécessitant une longue prise en charge et souvent assez coûteux. Des filles peuvent décider de partir du village alors qu'elles ne se sentent pas bien. Le voyage peut compliquer son état de santé, et dans ce cas, ses connaissances en ville peuvent la faire repartir chez ses parents. 


\section{Réseaux Sociaux en Ville}

Les réseaux sociaux des filles à Bobo-Dioulasso ne diffèrent pas de ceux dont elles ont fait partie au cours du voyage. Pour l'ensemble des filles, les personnes qu'elles rencontrent pendant les premières journées sont les tuteurs et les membres de la grande famille (tantes, oncles, grandfrère ou grande-sœur).

Les premières personnes rencontrées peuvent aussi être dans une moindre mesure les employeurs comme le précise cette fille.

"Il y a des gens en ville qui demandent des aides ménagères. A partir du village, leurs parents les appellent pour les informer de notre arrivée. De la gare, ces gens qui nous accueillent sont donc nos tuteurs"

-FILLE 11 ANS, EN PROVENANCE DE SOLENZO, 1 AN D’ACTIVITE.

\section{Circuit de recherche de domicile et de travail}

Dans la ville, la recherche de travail et de domicile repose régulièrement sur leurs parents (oncle, tante, frères ou sœurs). De l'avis de certaines filles, il y en a qui ne bénéficient plus de l'appui de leurs parents ou tuteurs pour trouver un travail en cas de comportements désobligeants notamment la survenue de grossesse, les mauvais comportements, etc. Une fille explique :

"Une fois en ville, c'est la tante qui aide à avoir du travail; mais une fois en cas de problèmes, comme par exemple si une fille tombe enceinte, la tante ne veut plus l'aider "

-FILLE DE 13 ANS, EN PROVENANCE D’ABIDJAN DEPUIS 2003.

Aussi note-t-on que certaines filles ont un travail assuré avant le départ du village. La responsabilité de trouver du travail incombe aussi aux filles elles-mêmes, qui vont de famille en famille. Cette situation est régulière chez les filles d'un âge avancé et ayant une connaissance de leur environnement de travail.

"Toi-même, tu cherches du travail. Tu peux aller de cours en cours pour demander du travail "

-FILLE DE 15 ANS ARRIVEE EN 2008.

"Moi, je descends chez mon tuteur et je cherche moi-même du travail "

-FILLE DE 16 ANS, ARRIVEE EN 2007, EN PROVENANCE DE DIEBOUGOU.

\section{Régularité et fréquence des visites aux tuteurs}

Après quelques mois d'activités, l'ensemble des filles affirment maintenir les relations avec ces mêmes personnes qui ont été présentes quand elles sont arrivées pour la première fois. Plusieurs raisons expliquent le maintien de ces relations : reconnaissance pour les avoir aidées à trouver du travail, visite en cas de problème, désir de leur confier leur argent à garder, etc.) Comme l'attestent ces propos:

"Quand le travail finit, je demande la permission au patron pour aller saluer la personne qui m'a trouvée du travail. II peut aussi arriver que tu ne travailles pas bien parce que tu es malade. En voyant que le patron n'est pas content parce que tu ne travailles pas, on peut aller voir le tuteur pour intervenir pour qu'il ne te renvoie pas".

-FILLE DE 13 ANS, EN PROVENANCE D’ABIDJAN, 2 ANS D'ACTIVITE. 
"Les filles voient généralement les premières personnes deux fois dans le mois pour remettre leur argent ou pour discuter des malentendus avec l'employeur "

-FILLE DE 16 ANS, 3 ANS D'ACTIVITE, ORIGINAIRE DE KAYA.

La fréquence des visites varie d'une fille à l'autre en fonction de la distance du lieu d'habitation du tuteur d'avec celui de la fille. La fréquence moyenne de visite est d'une fois par semaine. La fréquence de visite des filles dépend aussi des humeurs du patron comme le précise cette fille de 15 ans :

"Il y a des patrons qui n'aiment pas que les visites soient trop fréquentes. Ils pensent qu'on partira raconter ce que nous vivons à la maison chez nos tuteurs "

-FILLE DE 15 ANS, ORIGINAIRE DE BOBO, EN ACTIVITE DEPUIS 2 ANS.

\section{Relations avec le Village d'Origine}

Une fois en ville, les filles entretiennent des rapports avec les parents généralement par le biais du téléphone portable que certaines d'entre elles ont pu acquérir grâce à leurs revenus de travail.

Quelques filles sollicitent les téléphones de leurs tuteurs (trices) ou des parents pour effectuer les appels. Pour celles qui ne sont pas très loin de leur village, quelques filles gardent les relations avec le village en s'y rendant régulièrement.

"Je peux me rendre là-bas aussi pour les voir puisque le village n'est pas loin "

-FILLE DE 13 ANS, KOUMI (BOBO-DIOULASSO)

La régularité de contact est d'une fois par semaine pour la plupart des filles. Quelques filles effectuent un appel par mois ; d'autres le font en fonction de leur pouvoir financier.

"Moi, j'appelle la famille si j'ai des crédits (des unités de recharge)"

-FILLE DE 13 ANS, ORIGINAIRE D'ABIDJAN

Les filles reçoivent aussi des appels directement de leurs parents. Les personnes spécifiques contactées sont les membres de la famille immédiate. Le père et la mère sont régulièrement les personnes contactées et le cas échéant, la grande sœur ou le frère.

Les échanges au téléphone portent sur des sujets divers variables en fonction de l'actualité du moment. Dans la plupart des cas, le souci d'avoir des nouvelles de santé ou l'envie d'entendre les parents sont la motivation essentielle du maintien $d u$ contact avec la famille.

Par ailleurs, bien que petites et n'ayant pas assez de revenus financières, plusieurs autres filles restent en contact avec les parents pour les aider à résoudre certains problèmes comme l'attestent ces propos. (cf. encadré).
Les citations ci-dessous montrent que les filles contribuent aux dépenses de la famille en envoyant de l'argent à leurs parents en cas de besoin.

"Je reste en contact pour savoir si tout le monde se porte bien et s'il y a des difficultés comme le manque de nourriture ou d'argent; alors je leur envoie de l'argent " -FILLE DE 13 ANS, EN PROVENANCE DE COTE D'IVOIRE

"Je les appelle pour savoir quelles sont les nouvelles. S'il y a des problèmes d'argent, j'envoie leur donner " -FILLE DE 14 ANS, ORIGINAIRE DE BANFORA, 1 AN D'ACTIVITE

" Je leur demande quels problèmes ils ont. Je peux par exemple payer une charrette ou des habits ou un vélo pour les envoyer" -FILLE DE 15 ANS, VENANT DE SIBY, 1 AN D'ACTIVITES

"Ils peuvent me demander si j'ai de l'argent si oui, je paie quelque chose pour leur donner (savon, vélo, etc.)" ;

-FILLE DE 13 ANS, VENANT DE KOUPELA, 4 ANS D'ACTIVITE 
A l'inverse, les parents entretiennent des relations avec leurs filles, pour les inviter au retour en vue de se préparer au mariage. Fille de 16 ans, originaire de Safané explique :

"Comme on est venu pour travailler, on peut durer ici. Donc, les parents peuvent nous appeler pour dire qu'on a eu un mari et qu'il faut qu'on revienne pour nous préparer au mariage ${ }^{1 "}$.

\section{Occupations des Jeunes Filles et Relations Sociales en Ville \\ Occupation des filles pendant les moments libres}

En dépit de leur programme chargé, les filles disposent de jours de repos (les dimanches) au cours desquels elles rendent visite à leurs proches. La majorité des filles rencontrées passent ces moments avec leurs parents originaires du même village qu'elles. A leurs moments libres, elles passent une partie du temps chez leurs copines de même âge.

"Le jour où je ne travaille pas, j'informe mon patron que je sors et je vais chez mes copines"

-FILLE DE 13 ANS, EN PROVENANCE D’ABIDJAN, 3 ANS D'ACTIVITE

Leur temps de repos constitue leur temps libres et elle profite, ou pour rendre visite à leurs parents au village. Ce cas parait assez régulier pour les filles dont le village est situé en moyenne à $100 \mathrm{~km}$ de Bobo-Dioulasso (Diébougou, Banfora). Le retour chez l'employeur a lieu le soir comme l'atteste cette fille :

"Le jour que je ne travaille pas je rends visite aux parents et y passe la journée et revenir le soir"

-FILLE DE 16 ANS, ORIGINAIRE DE KAYA.

Les jours de repos ou les quelques temps libres (surtout en cas d'absence du patron) sont utilisés pour venir en aide à leurs copines dans les travaux ménagers (lessive surtout) :

"Je pars aider une amie si je n'ai rien à faire "

-FILLE DE 17 ANS, ORIGINAIRE DE DIEBOUGOU, 9 MOIS D’ACTIVITE.

\section{$\underline{\text { Relations sociales des filles en ville }}$}

D'une manière générale, les réseaux sociaux des filles en ville sont créés principalement avec les parents. Les rencontres s'effectuent à leur domicile ou dans les marchés.

"Au marché on voit quelques personnes venues aussi du village pour travailler en ville"

-FILLE DE 15 ANS, EN PROVENANCE DE YAKO, 3 ANS D'ACTIVITE.

Les filles entretiennent des relations animées avec leurs copines de même âge dans les marchés, ou dans les quartiers. Les rencontres sont régulières mais leur fréquence peut varier

\footnotetext{
${ }^{1}$ Au Burkina Faso, la pratique du mariage précoce chez les jeunes filles est toujours répandue avec des fortes proportions dans certaines régions. Selon les données de l'EDS 2003, près de $52 \%$ des filles de $20-24$ ans mariées avant l'âge de 18 ans et $5.2 \%$ se sont mariées avant l'âge de 15 ans.
} 
en fonction du lieu d'habitation de chaque fille. Souvent, les rencontres sont hebdomadaires ou mensuelles. Pour d'autres filles, la régularité des rencontres se fait à plusieurs fois par semaine.

Pour quelques filles dont les lieux d'habitation sont proches les unes des autres, les rencontres sont fréquentes mais brèves comme relaté à travers ces propos :

"S'il n'y a pas de travail, on peut se voir souvent, plusieurs fois dans la journée dès que possible"

-FILLE DE 16 ANS, EN PROVENANCE DU MALI, 3 ANS D’ACTIVITE.

"On peut se voir plusieurs fois dans la journée si nos cours (habitations) ne sont pas loin l'une de l'autre. De fois, on peut se voir plusieurs fois dans la journée ; elle passe me voir à tout moment si elle finit de travailler. Si moi aussi je finis, je vais l'aider "

-FILLE DE 13 ANS VENANT DE COTE D'IVOIRE, EN ACTIVITE DEPUIS 3 ANS.

D'une manière générale, au cas où les contacts fréquents entre les filles n'entravent pas le travail, la plupart des employeurs n'y trouvent pas d'inconvénients. II reste entendu que par mesure de sécurité, certains employeurs contrôlent les fréquentations des filles.

Le contenu de discussion lorsque les filles se retrouvent entre elles

"On échange aussi sur comment nous garder surtout du côté des garçons "

-FILLE DE 16 ANS, EN ACTIVITE DEPUIS 9 MOIS, EN PROVENANCE DE DIEBOUGOU.

"On se donne des conseils, pour ne pas prendre des grossesses et de ne pas utiliser les objets souillés " -FILLE DE 13 ANS, EN PROVENANCE D'ABIDJAN, 3 ANS D'ACTIVITE.

"Les filles se donnent des conseils concernant les garçons, les contraceptifs et d'autres choses pour éviter les maladies et sur l'hygiène corporelle et alimentaire " -FILLE DE 16 ANS, EN ACTIVITE DEPUIS 3 ANS.

"On peut parler de nos patrons. Surtout si ce qu'ils font n'est pas bien "

-FILLE DE 16 ANS, EN ACTIVITE DEPUIS 4 ANS.

"Mon amie peut venir me voir et me donner des conseils et dire de ne pas prendre ce que mon patron ne m'a pas donné. On parle des employeurs quand il y a un problème "

-FILLE DE 16 ANS, EN ACTIVITE DEPUIS 2 ANS.

\section{Sujets abordés entre les filles}

Au cours de leurs rencontres, ces filles abordent des sujets relatifs selon elles aux nouvelles de leurs parents au village.

"Les filles parlent de leur village et aussi de leur famille"

-FILLE DE 12 ANS, 2 ANS D'ACTIVITE

Ces nouvelles qu'elles partagent entre elles sous forme introductive font place à d'autres sujets relatifs aux meilleures manières de vie pour une fille de leur âge et travaillant dans des ménages. Ainsi les sujets abordés font office de conseils mutuels dans le domaine de la santé, la 
lutte contre les grossesses non désirées, les comportements à éviter dans les ménages, I'hygiène corporelle et pendant les règles, etc. On constate que les sujets de discussion des filles portent sur les thématiques abordées lors des sessions hebdomadaires. C'est dire qu'au-delà des rencontres formelles, les filles partagent ce qu'elles apprennent dans le programme "Filles Eveillées" avec d'autres camarades dans leur entourage.

\section{Attitudes en cas de conflits avec l'employeur}

Les cas de malentendus n'ont pas été clairement évoqués par les filles rencontrées au cours des focus. Toutefois, lorsqu'ils surviennent celles-ci font recours aux parents, à leurs tuteurs ou à tout autre intermédiaire à mesure de les aider à demander des excuses auprès de leurs patrons.

"Je vais voir une personne âgée qui intervient. Toute personne qui connait la honte et qui peut nous aider peut intervenir à notre faveur "

-FILLE DE 17 ANS, ORIGINAIRE DE SAFANE, 3 ANS D'ACTIVITE.

"Les grandes sœurs peuvent aussi intervenir. Elles vont dire que ce sont des enfants et qu'il ne faut pas que les patrons se fâchent beaucoup "

-FILLE DE 15 ANS, EN PROVENANCE DE SIBY, 1 AN D'ACTIVITE.

Cette attitude anime aussi certaines filles qui estiment que le patron même est à l'origine du malentendu. Quelques filles interviennent directement auprès du patron, mais ces cas sont rares.

Ainsi, les propos relatés par les filles montrent qu'en cas de conflits, elles ne sont pas toujours en première ligne dans la résolution de leurs problèmes. Du reste, les mentors ou les chargés de programme de l'Association Tié participent dans certains cas à la résolution de ces problèmes.

\section{Attitudes en cas de rupture de contrat}

Dans le contexte de travail au niveau des ménages, les ruptures de contrat sont légions et peuvent émaner de la volonté des deux parties (soit sur désir de la fille ou au contraire de la volonté du patron). Dans ce contexte, de l'avis de la majorité des filles, le premier secours sollicité est l'aide du tuteur. Celui-ci les héberge et les aident ensuite à trouver un autre travail.

\footnotetext{
"La fille se dirige vers la personne qui l'a amenée du village (le tuteur ou le parent) " fille de 11 ans, originaire de Solenzo, 2 ans d'activité

"Pour trouver du travail, je peux aller chez d'autres parents qui vont s'occuper de moi et m'aider à trouver du travail."
} 
Pour celles qui n'ont pas du tuteur, ou n'ayant pas gardé des liens avec ces derniers, elles font recours à leurs copines qui les reçoivent sur autorisation du patron de ces dernières.

"La fille peut se rendre chez son amie qui va en parler à son patron. S'il n'est pas compliqué, elles vont dormir ensemble là-bas. Elles vont ensuite, commencer à chercher du travail, jusqu'à ce que la fille ait du travail "

-FILLE DE 13 ANS, EN PROVENANCE D'ABIDJAN, 3 ANS D'ACTIVITE.

"De fois, c'est le patron de sa camarade chez qui elle dort qui l'aide aussi à trouver du travail"

-FILLE DE 13 ANS, ARRIVEE DEPUIS 2007.

Par ailleurs, d'autres filles décident de rentrer au village, où elles peuvent rester ou revenir de nouveau en ville. Dans ce cas de figure, plusieurs d'entre elles (les filles d'un âge avancé) partent pour Ouagadougou, la capitale, dans l'espoir de trouver un travail plus décent et rémunérateur.

On note que les recours des filles en cas de problème se limitent aux personnes qu'elles fréquentent habituellement que sont les amies, les tuteurs ou les parents. II est donc important d'élargir les possibilités de recours des filles en cas de problèmes en leur fournissant des contacts des structures privées ou publiques de référence dans la communauté, comme on fait dans le contexte du programme "Filles Eveillées".

\section{Capacités Financières}

Derrière les durs labeurs entachés quelques fois d'humiliation au niveau de certains ménages, se cachent une forte volonté de gagner de l'argent pour la résolution de leurs propres problèmes, mais aussi ceux de leurs parents. Cette investigation a permis de savoir si les filles atteignent leurs objectifs ou quels sont les défis en matière de gestion de leurs ressources financières.

\section{L'Utilisation de Leurs Revenus}

A la question de savoir ce qu'elles font en général de l'argent qu'elles gagnent en travaillant, l'ensemble des filles répondent qu'une partie de l'argent est dans un premier temps utilisée pour l'achat de trousseau de mariage composé d'effets d'habillement, d'ustensiles de cuisines. Une autre partie de leurs revenus est envoyée aux parents au village et doit servir à l'achat de compléments alimentaires, ou pour honorer leurs frais d'ordonnance en cas de problème de santé.

"On envoie une partie de l'argent au village. On peut dire que tu as eu un mari ; donc on peut acheter beaucoup de choses. Tu en parle à ton patron qui te donnes ton argent et tu paies tes affaires de mariage)"

-FILLE DE 13 ANS, ORIGINAIRE DU MALI, 3 ANS D'ACTIVITE, NIVEAU DU CM2.

"On envoie au village, si nos parents sont malades, on les aide à se soigner "

-FILLE DE 13 ANS, EN PROVENANCE D'ABIDJAN, 3 ANS D'ACTIVITE.

Au-delà des soucis de la constitution du trousseau de mariage et ceux de participer à la résolution de problèmes du village, les filles ont noté la nécessité pour elles de garder de l'argent 
pour plusieurs raisons : se soigner en cas de maladie, développer des activités génératrices de revenus dans l'avenir, etc.

"On paie des affaires de femmes; mais, on ne dépense pas tout. On peut se soigner si on est malades ou bien si un problème survient, on peut le résoudre "

-FILLE DE 16 ANS, 3 ANS D'ACTIVITE, ORIGINAIRE DE BAMAKO.

"Je prépare mon avenir et épargne un peu pour faire face aux maladies et autre problèmes"

-FILLE DE 16 ANS, ORIGINAIRE DE PADEMA, BOBO, 2 ANS D'ACTIVITE

\section{Capacités de Filles à Épargner Leurs Revenus Financiers}

Toutes les filles affirment ne pas dépenser tous leurs revenus pour des raisons déjà évoquées dans les paragraphes antérieurs (cf. paragraphe 3.1). Le système d'épargne courant évoqué par la majorité des filles consiste à confier l'argent à des parents, aux tuteurs ou à l'employeur qu'elles recontactent en cas de besoin.

"Je peux garder l'argent chez moi, ou bien, je dis à mon patron de garder jusqu'à ce que j'en aie besoin. Ou encore je peux aller donner à quelqu'un d'autre de déposer "

-FILLE DE 17ANS, ORIGINAIRE DU SUD-OUEST, 9 MOIS D'ACTIVITE.

Pour quelques-unes des filles, le mode d'épargne s'effectue à travers les tontines ${ }^{2}$ en collaboration avec leurs tiers.

Ces pratiques comportent des risques pour les filles notamment le non remboursement de l'argent lorsqu'elles en auront besoin. D'où la nécessité de renforcer leurs compétences à recourir aux établissements financiers, mais qui malheureusement ne sont pas souvent accessibles aux filles ou ont des conditions pas favorables. Au Burkina Faso, l'âge minimum pour ouvrir et pouvoir faire des opérations dans une banque est de 21 ans. On peut ouvrir un compte bancaire pour quelqu'un qui n'a pas 21 ans mais le compte sera sous autorité parentale.

Les enquêtes ont monté que l'épargne à travers les établissements financiers (caisses d'épargne et les banques) n'est pas connu de toutes les filles ou que les conditions ne sont pas accessibles ou intéressantes. Les raisons de ce non intérêt sont la non importance des sommes qu'elles épargnent, la méconnaissance du milieu, la retenue des frais de gestion par la banque, et leur jeunesse.

"Je ne vais pas à la banque, parce qu'elle fait des retenus sur notre argent (frais de gestion du compte)"

-FILLE DE 13 ANS, ORIGINAIRE D'ABIDJAN.

"Certaines filles ont trop de problèmes. Tu déposes en caisse et à cause des problèmes, tu viens chercher rapidement. A cause de ça, on ne vaut pas aller à la caisse "

-FILLE DE 15 ANS, NIVEAU DU CM2.

Le fait que les filles soient migrantes constitue également un obstacle pour elles de recourir à ces institutions, surtout qu'elles n'ont pas de personnes qui peuvent les aider à accéder aux

\footnotetext{
${ }^{2}$ Ce système consiste à participer à des cotisations mensuelles sur la base d'un montant fixe. Le montant rassemblé du mois est donné à chaque membre à tour de rôle.
} 
services bancaires. Elles ne pourront pas effectuer des opérations lorsqu'elles seront au village, donc elles pensent que ce n'est pas nécessaire d'épargner dans une banque.

"On vient dans la grande ville, et on ne dure pas souvent. Tu fais seulement deux jours et tu dois repartir. Donc pour moi, ça ne sert pas [d'ouvrir un compte d'épargne] "

-FILLE DE 17 ANS, NIVEAU D'ETUDE DU COURS PREPARATOIRE. 1ERE ANNEE, ORIGINAIRE DE DIEBOUGOU

"Dans les grandes villes, on ne connait pas quelqu'un. Et puis, il faut attendre un moment pour aller prendre. Souvent ce moment n'arrive pas et nous on a besoin d'argent de fois tout de suite "

-FILLE DE 16 ANS, NIVEAU DU CM2.

\section{Défis en Matières de Gestion de Leurs Ressources Financières}

La majorité d'entre elles affirment que le circuit de la banque et des caisses d'épargne offrent plus de garantie en matière de sécurité financière bien qu'elles soient inaccessibles.

L'épargne chez des tierces personnes n'est pas de ce fait appréciée par ces filles en raison des multiples problèmes qu'elles rencontrent pour entrer en possession de leurs ressources financières.

"Tu peux donner l'argent au patron. Mais s'il y a des problèmes il va le dépenser. Et quand tu en as besoin pour résoudre un problème, tu vas lui dire et il va te tourner pendant longtemps. Yé !! Le fait de donner l'argent aux gens pour qu'ils déposent n'est pas facile. Ce qui est sûr, il y aura trop de paroles. Cette personne dira qu'il a eu un problème et que qu'il a dépensé l'argent, mais qu'il va rembourser."

-FILLE DE 14 ANS, NIVEAU DE CM2, 1 AN D'ACTIVITE.

"Avec le patron quand tu as besoins de ton argent, il va te dire de venir lundi, ensuite, te dire de venir un autre jour, et ainsi de suite. Donc quelques fois, il y a problème "

-FILLE DE 13 ANS, 1 ANNEE D’ACTIVITE.

"D'autres peuvent dire qu'ils ont été victime de vol et que le voleur est parti avec l'argent en plus de la télé "

-FILLE DE 15 ANS, DU NIVEAU DE CM2.

"De fois, le patron ou le tuteur dure avant de te donner l'argent; ta maman peut t'appeler de venir et tu es obligée de prendre un crédit pour aller au village. Souvent, il m'arrive de pleurer à cause de ça"

-FILLE DE 15 ANS, 9 MOIS D'ACTIVITE.

Pour d'autres filles l'implication des parents directs dans l'épargne de leur argent ne présentent pas de risque. II en est de même pour certains employeurs qui ne manifestent aucun intérêt à utiliser l'argent.

\section{Opinions par Rapport à un Appui en Matière de Gestion des Revenus}

A la lumière des risques liés au mode de gestion de leurs revenus par de tierces personnes, l'ensemble des filles approuvent l'idée d'un éventuel appui dans ce domaine. Elles souhaitent 
donc qu'on puisse aider les filles employées de maison à épargner dans les établissements financiers. De leurs avis, cela contribuera à garantir une meilleure sécurité financière. En outre, l'épargne par le circuit des banques peut contribuer à une meilleure régulation des dépenses.

"[Les banques] c'est bien parce que si j'ai besoin de l'argent c'est facile. L'argent est en sécurité"

-FILLE DE 16 ANS, EN ACTIVITE DEPUIS 3 ANS.

"Epargner en banque nous aide dans la bonne gestion et soutenir les parents en cas de maladie"

-FILLE DE 16 ANS.

"Je souhaite avoir un autre travail que le travail d'aide-ménagère pour mieux se prendre en charge dans l'avenir. Lorsqu'on a des revenus plus importantes, on sera obligé de mieux gérer en cherchant à aller dans les banques"

-FILLE DE 16 ANS, 2 ANS D'ACTIVITE.

\section{CONCLUSION}

Cette étude sur les réseaux sociaux et les capacités financières met en relief le rôle des aides ménagères dans la gestion de leur propre argent, la gestion de l'économie des ménages au niveau des sites d'accueil (Bobo-Dioulasso) mais surtout dans leur famille d'origine. De prime abord, ces départs quelquefois voulus par la famille s'effectuent de manière linéaire sans lieu de transit. Les filles sont dans leur grande majorité accompagnées par des parents, et dans une moindre mesure par des tuteurs, dont elles sont dépendantes tout au long du voyage mais aussi une fois en ville. Du reste, elles maintiennent les liens avec ces parents ou tuteurs qu'elles rendent visite souvent de manière hebdomadaire. Ceux-ci sont par ailleurs sollicités pour la recherche du travail, mais aussi en cas de problèmes avec leurs employeurs. Les filles maintiennent aussi des liens étroits et réguliers avec leurs familles du village, à qui elles apportent du soutien financier.

Les investigations montrent que leurs copines de même âge travaillant dans les ménages forment les membres de leurs réseaux. Entre elles, les visites sont régulières soit à leurs domiciles respectifs, soit dans les lieux de vente (marchés ou quartiers). Aussi note-t-on que ces réseaux représentent des circuits d'informations des filles. Les occasions de rencontre se transforment en cadre d'éducation et de conseils en vue d'éviter les pièges ou des situations malheureuses en matière de santé ou chez l'employeur.

Dans un cas comme dans l'autre, il arrive que les filles soient confrontées à de nouvelles situations (séparation d'avec l'employeur notamment). Dans ce contexte, les parents, tuteurs ou les copines soient sollicités pour des appuis divers (logement, assistance financière, etc.) dans l'attente d'une nouvelle situation plus propice.

Mais comme le relatent la majorité des filles au cours des focus, la mobilisation des ressources financières par le travail constitue le principal souci des aide-ménagères. L'argent mobilisé est généralement réparti entre l'achat du trousseau de mariage, l'envoi à la famille au village et le placement chez une connaissance. Dans le dernier cas, les filles rencontrent plusieurs problèmes (difficulté de rentrer en possession de leur argent). Cela est la base du 
découragement et de situations conflictuelles, occasionnant l'intervention de tierces personnes (tuteurs ou autres connaissances).

Les questions de gestion harmonieuse des revenus constituent une préoccupation pour l'ensemble des filles qui ne connaissent pas les circuits d'épargne courants (caisse d'épargne, banques) ou qui les trouvent non accessibles.

\section{RECOMMENDATIONS}

Ces discussions de groupe relatent un ensemble de difficultés qui débouchent sur des suggestions et recommandations :

* Etant donné que le réseau social des filles migrantes reste beaucoup plus limité aux membres de la famille, les programmes en leur direction devraient mettre l'accent sur la création de réseaux sociaux avec des amies de même âge. Le programme "Filles Eveillées " y apporte une réponse à travers le modèle basé sur les trois éléments clés du programme (espace sûr, amies, mentor).

* Donner les moyens techniques aux filles qui participent au programme de partager ce qu'elles apprennent lors des sessions éducatives avec leurs camarades en renforçant par exemple leur compétence en technique de communication. L'expérience a montré que les filles du programme discutent de ce qu'elles apprennent lors des sessions, avec les autres copines dans les quartiers. Dans le programme "Filles Eveillées" il est prévu l'élaboration d'une boîte à images très simple qui pourra être utilisée par les filles qui ont participé au programme de retour dans leur village d'origine pour partager les compétences et les enseignements reçus à leurs camarades et leurs sœurs et ça doit être considéré dans le contexte de la ville aussi.

* En même temps, encourager et accompagner les filles employées de maison à utiliser les établissements financiers ; L'expérience a montré que même si les filles savent où trouver une institution financière, elles n'ont pas souvent le courage d'y aller par méconnaissance des conditions d'accessibilité. Cette crainte est accentuée chez les filles non alphabètes.

* Au regard des difficultés mentionnées, un plaidoyer devrait être fait au plus haut niveau afin que les institutions financières soient accessibles aux adolescentes sans la nécessité d'avoir une garantie. Elles font un travail qui leur procure une rémunération périodique et c'est la première fois pour la plupart d'entre elles de gérer de l'argent. Le renforcement de leurs compétences en capacités financières s'avère nécessaire pour les aider à atteindre leurs objectifs (acheter des effets personnels et épargner de l'argent).

* Sensibiliser les filles sur les risques de l'épargne traditionnelle de leurs revenus (tontines). L'expérience a montré que l'épargne traditionnelle comporte trop de risques surtout pour les filles adolescentes: le risque de ne pas percevoir son argent car cela dépend de la moralité des membres du groupe. 
* Développer un partenariat avec les structures simples d'épargne et légales à mesure d'accompagner les filles. Les caisses populaires pourraient être une opportunité car elles ont une couverture de représentations géographiques plus large que les autres institutions. Ils ont des représentations souvent mêmes dans les petites provinces. Cela pourrait être une opportunité pour ces adolescentes employées de maison qui sont très mobiles et qui mènent un travail saisonnier. 